\title{
Verifying the Geometric Accuracy of Sentinel-1A and Sentinel-1B using L0- Products
}

\author{
Kersten Schmidt ${ }^{1}$, Nuria Tous Ramon ${ }^{1}$, Jens Reimann ${ }^{1}$, Marco Schwerdt ${ }^{1}$ \\ ${ }^{1}$ Microwaves and Radar Institute, German Aerospace Center (DLR), Oberpfaffenhofen, Germany, kersten.schmidt@dlr.de
}

\begin{abstract}
The geometric accuracy of Sentinel-1 is usally derived from level-1 products in Stripmap mode using accurate surveyed corner reflector positions. While the range offset is found to be low $(\sim 30 \mathrm{~cm})$ an azimuth offset of about $2 \mathrm{~m}$ remains. The study investigates the reason for the geometric offsets by deriving the geometric parameters from L0-data. Therefore the pulse round-trip time is analyzed from transmission to reception of a point target's backscatter including a simple pulse compression in range and azimuth. Finally, the azimuth offset is reduced to about $15 \mathrm{~cm}$ which indicates an artificial bias arising from SAR processing.
\end{abstract}

Index Terms - antenna, propagation, measurement.

\section{INTRODUCTION}

Synthetic aperture radar (SAR) images are commonly used for Earth observation; in contrast to optical systems, SAR instruments can be operated more independently from temporal (day or night) and meteorological conditions. Since the spatial resolution of SAR images has been improved also for space-borne missions to accuracy values in the order of meters and sub-meters at both, range and azimuth direction $[1,2]$, the geocoding of related products plays also an important role. In general, additional parameters or look-up tables are delivered in combination with SAR data products to allow the association of any pixel in a given SAR image with its geographical position on the ground.

The Sentinel-1 mission in the frame of ESA's COPERNICUS program aims to ensure long-term Earth observation under stable conditions. Both satellites Sentinel1A (S-1A) and Sentinel-1B (S-1B) are operated in a near polar, sun-synchronized orbit, each with a repeat cycle of 12 days in such a way that the two satellite constellation offers a 6-day repeat cycle over a given observation area. Both SAR instruments operate at C-band $(5.405 \mathrm{GHz})$ and carry a rightlooking active phased array antenna. Dual polarization operation $(\mathrm{HH}+\mathrm{HV}, \mathrm{VV}+\mathrm{VH})$ is realized by two parallel receive chains. Sentinel-1 provides different operation modes, but in the current study only Stripmap mode is evaluated. Six different Stripmap beams are available each with an individual elevation range, but all with a similar swath coverage of $80 \mathrm{~km}$ and a spatial image resolution of $5 \mathrm{~m} \times 5 \mathrm{~m}[3,4]$.
The geolocation accuracy of space-borne SAR instruments also known as pixel localization accuracy has to be determined and verified by analyzing SAR images. To achieve and verify a high spatial accuracy, below one meter, the related geometric conditions have to be known very accurately, e.g. spacecraft orbit data, the SAR antenna geometry related to the spacecraft's center of mass and the reference points on Earth. Moreover, SAR processing has to consider all relevant timing issues arising during range and azimuth focusing, e.g. the instrument internal delay or issues arising from interpolation, approximation and signal filtering techniques. Furthermore, the wave propagation is affected by the atmosphere; considerable delays are induced by the troposphere and ionosphere and need to be considered accordingly.

The pixel localization accuracy of both S-1A and S-1B was analyzed during their respective commissioning and routine operation phases within several studies $[5,6,7,8]$ by evaluating SAR images (L1 products) processed with the Sentinel-1 operational SAR Instrument Processing Facility (IPF). In particular for Stripmap mode, standard deviations for the absolute location error in range and azimuth remain low below $0.5 \mathrm{~m}$. But for all four studies an offset in azimuth of about $2 \mathrm{~m}$ was found; in all cases, the azimuth time derived from the SAR data headers precedes the predicted overpass time given by precise orbit data. The reason for this bias could arise from an inaccurate SAR processing or from non-considered additional geometric offsets like a significant distance between the spacecraft center of mass and the SAR antenna phase center.

In order to address this issue, this paper proposes a new and easy applicable method to verify the geometric accuracy based on SAR raw data (L0) analysis. The method analyzes the timing information from the L0 raw data headers and compares it to the target responses of precisely surveyed point targets before (an explicit) SAR processing is performed. The method includes simplified range and azimuth compression procedures and focuses mainly on selected data which contain the target response. 


\section{METHOD}

In order to verify the geometric properties of the SAR products, the signal propagation path has to be completely retrieved: from transmitting the SAR pulses up to receiving the backscatter power from the point target. The main parameters to be considered are depicted in Fig. 1: the geometry between spacecraft and point target is determined by the orbit state vectors and the point target position on Earth. The information about transmitted and received pulses is saved by the SAR instrument. Along the propagation path the pulses are affected by additional effects (mainly due to the troposphere and the ionosphere).

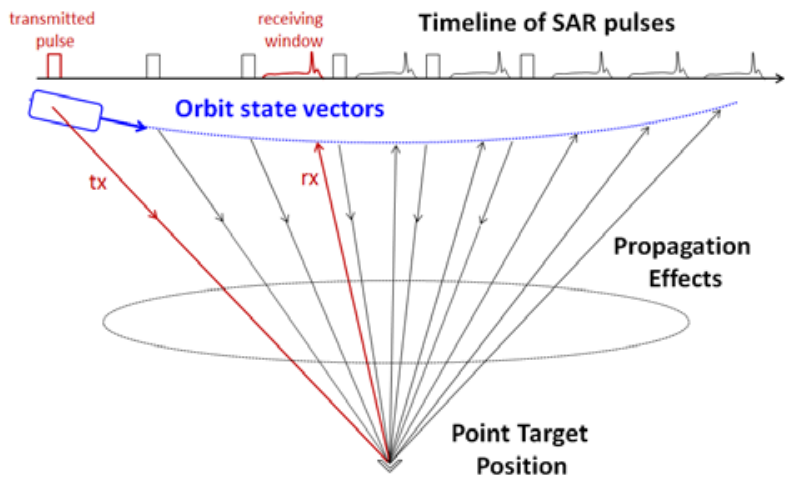

Fig. 1. SAR observation scheme containing main parameters which have to be considered for the geometric retrieval: the orbit state vectors and the target position define the geometry between spacecraft and target; the pulse timeline stored in the raw data headers contains information about transmitted (tx) and received (rx) SAR pulses.

\section{A. Range Direction}

The pulse transmitted by the Sentinel-1 SAR instruments is a chirp signal. The received signal is acquired within the receiving window between two transmission pulses. In order to extract the impulse response of the target from the L0 data, the received signal is correlated with the ideal chirp (match filter); the output gives the range compressed signal for the full range line. A high radar backscatter due to a transponder or corner reflector is easy detectable within the range compressed signal as seen for the corner reflector response in Fig. 2 at $131.84 \mu \mathrm{s}$.

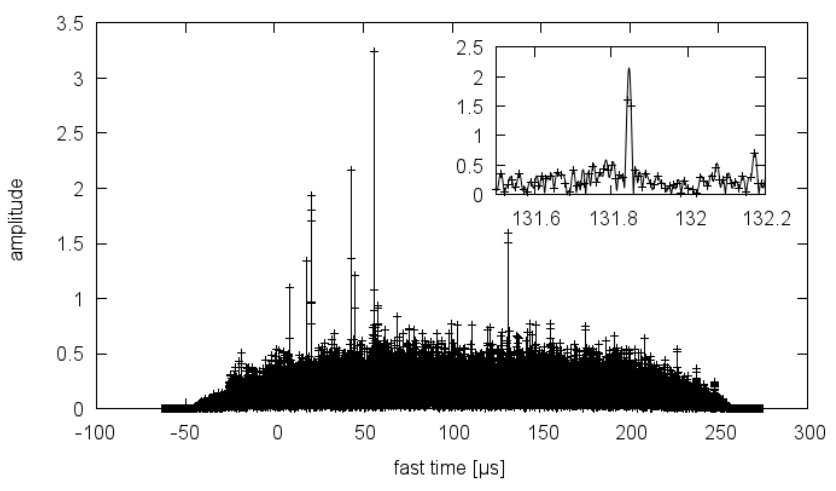

Fig. 2. Range compressed data of a single range line containing the target response of a corner reflector at $131.84 \mu \mathrm{s}$ fast time. The origin (fast time = 0 ) corresponds to the start of the receiving window; artificial data points for a negative fast time results from the match filter procedure.
The fast time, seen in Fig. 2, is related to the start of the receiving window; for estimating the propagation time also the transmit time of the related pulse has to be taken into account. This measured signal propagation can be compared with the estimated propagation path length derived from the geometry divided by the speed of light. For the remaining time difference, the range delay offset, additional effects have to be considered, mainly the internal delay of the SAR instrument itself, and the atmospheric delay caused by the troposphere and ionosphere. The atmospheric corrections can be derived from the zenith path delay (ZPD) and the total electron content (TEC), both measured by nearby GNSS stations.

\section{B. Azimuth Direction}

The phase history of a specific target can be traced during an overpass. The unwrapped phase from the SAR L0 data is compared with the predicted phase derived from the geometry using orbit data. This is done by converting the propagation time $(\tau$ propagation $)$ between satellite and reference target into a phase value using the radar frequency $\left(\mathrm{f}_{\text {radar }}\right)$ as given in equation (1).

$$
\varphi=-\tau_{\text {propagation }} \cdot 2 \pi \cdot \mathrm{f}_{\text {radar }}
$$

Fig. 3 shows the phase history of pulses reflected by a point target for a single overpass. The observed SAR data phase shown in blue is not yet unwrapped with values between $-180^{\circ}$ and $180^{\circ}$; the predicted phase (red) is overlaid presuming the same phase offset at $\mathrm{t}=0$. After unwrapping the SAR data phase and comparing it with the predicted one obtained from the geometry, a low difference below $5^{\circ}$ to the geometric phase remains. This indicates that the azimuth timing between SAR instrument and used geometry is well harmonized; no significant azimuth offset is expected after azimuth compression.

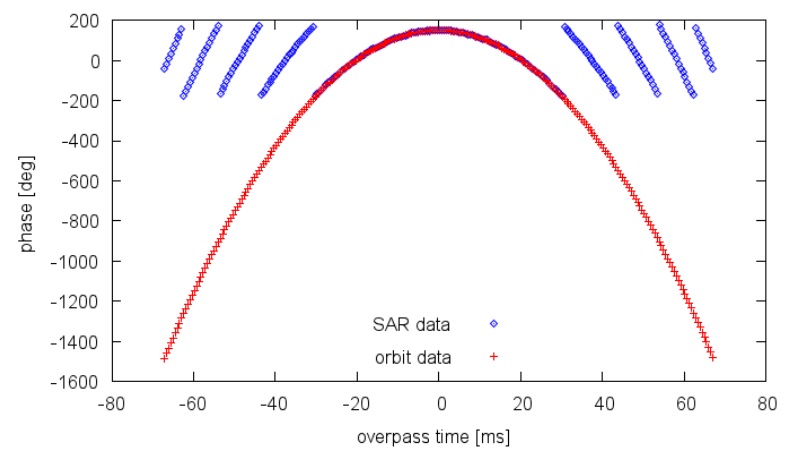

Fig. 3. Point target phase history derived from SAR data (wrapped phase in blue) and predicted phase from geometry (red). Each (blue) pixel represents the phase of the target response (amplitude peak within the range line) from an individual pulse received by the SAR instrument.

By correlating the measured phase history with the predicted one from geometry, the actual azimuth offset can be derived. The time at the correlation peak presents the azimuth shift which can be directly converted to an azimuth offset (in meters) by multiplying with the satellite velocity on ground. 


\section{RESULTS}

\section{A. Observation Period}

Two dedicated calibration campaigns were performed with Stripmap mode acquisitions using the corner reflectors of the DLR calibration site: between August and October 2015 and between December 2016 and February 2017, for S$1 \mathrm{~A}$ and S-1B, respectively (see Table 1). In case of S-1A, only the Stripmap beams S1, S3, and S6 were acquired but for both orbit directions (ascending and descending). For S1B all six Stripmap beams were acquired (S1 to S6) but each one of them either with ascending or descending orbit direction.

TABLE I. STRIPMAP OBSERVATION PERIOD OVER THE DLR CALIBRATION SITE

\begin{tabular}{|l|c|c|}
\hline \multicolumn{1}{|c|}{ Satellite } & S-1A & S-1B \\
\hline $\begin{array}{l}\text { Observation } \\
\text { Period }\end{array}$ & Aug 2015 - Oct 2015 & Dec 2016 - Feb 2017 \\
\hline & S1 - ASC & S1 - DES \\
Acquired Beams / & S1 - DES & S2 - ASC \\
Orbit Direction & S3 - ASC & S3 - DES \\
& S3 - DES & S4 - ASC \\
& S6 - ASC & S5 - DES \\
& S6 - DES & S6 - ASC \\
\hline
\end{tabular}

\section{B. Reference Target Contributions}

As reference targets three remotely controlled and configurable corner reflectors of the DLR calibration site located in Southern Germany were used [9]. For a given overpass each target is automatically aligned using an individual schedule configured from a remote station. The phase center location for each overpass is calculated from a model; the localization accuracy of the reference targets is verified to be below $3 \mathrm{~cm}$.

In addition to the propagation path derived from the geometry, further delays have to be considered: the internal delay of the SAR instrument itself, and atmospheric effects from the troposphere and ionosphere. While the internal delay is derived from calibration pulses performed within the SAR instrument, the delays due to the atmosphere are estimated from stations of the EUREF Permanent GNSS Network.

\section{Range Offset from Corner Reflectors}

The determined range offset from L0 data is depicted in Fig. 4 (red) for both satellites (S-1A: left and S-1B: right). In addition also the range offset from the L1 products are displayed (blue) which were already presented in previous studies $[7,8]$. An averaged range offset of $-0.99 \mathrm{~m}$ remains for the S-1A L0 products and of $1.05 \mathrm{~m}$ for the S-1B ones. These results match the offset between spacecraft center of mass and SAR antenna phase center which is the order of $1 \mathrm{~m}$ and not considered for the L0 evaluation. After compensating theses offsets, the remaining standard deviation is a measure for the pixel localization in range, which is $12 \mathrm{~cm}$ for S-1A and $6 \mathrm{~cm}$ for S-1B.

The range offsets derived from L1 products are smaller than the ones derived from L0 products. There is though one exception: the S-1B Stripmap beam S1 shows a larger offset with $1.6 \mathrm{~m}$ and indicates an inaccurate set of SAR processing parameters for this beam. The difference in range offsets derived from L0 and L1 products is in average of $1.3 \mathrm{~m}$ for $\mathrm{S}-1 \mathrm{~A}$ and of $1.2 \mathrm{~m}$ for S-1B (excluding the S1 beam results). Furthermore, the variation of the range offset for L1 products is nearly identical to the one derived from L0 products. Although no explicit indication is found in the L1 product annotation files, the small values of the L1 derived range offsets evidence that the distance between spacecraft center of mass and SAR antenna phase center is already considered during SAR processing.
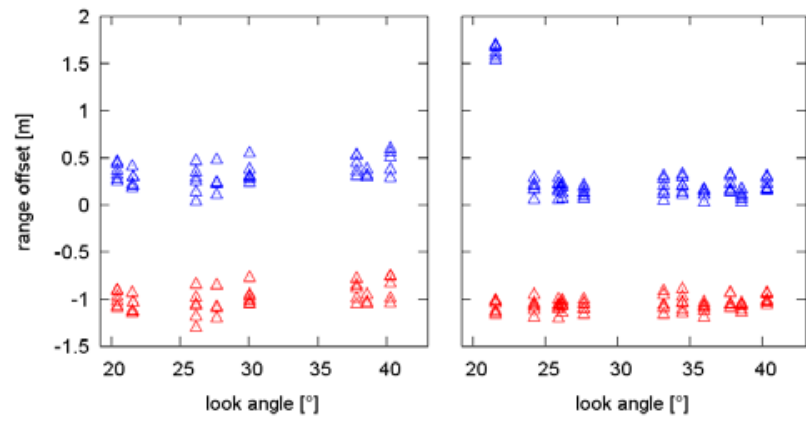

Fig. 4. Range offset derived from corner reflector responses for S-1A (left) and S-1B (right) obtained using both product types: L0 products (red) and $\mathrm{L} 1$ products (blue).

\section{Azimuth Offset from Corner Reflectors}

The derived azimuth offset is depicted in Fig. 5 using L0 data (red points) for both satellites (S-1A: left and S-1B: right). Similar to the range results also the azimuth offset derived from L1 products are shown (blue points). For the L0 products a very small azimuth offset remains, with $-13 \mathrm{~cm}$ in average for $\mathrm{S}-1 \mathrm{~A}$ and $-17 \mathrm{~cm}$ for $\mathrm{S}-1 \mathrm{~B}$. After compensating these offsets, the standard deviation is a measure of the pixel localization accuracy in azimuth which is derived from $\mathrm{L} 0$ products to $14 \mathrm{~cm}$ for both satellites.
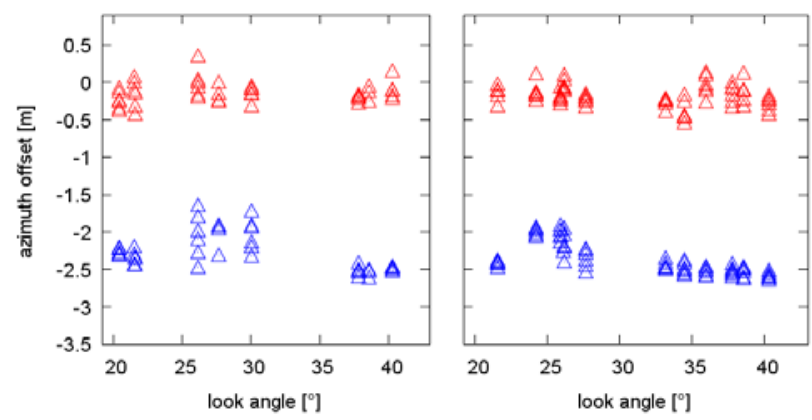

Fig. 5. Azimuth offset derived from corner reflector responses for S-1A (left) and S-1B (right) obtained using both product types: L0 products (red) and $\mathrm{L} 1$ products (blue).

In contrast to these results, the analysis performed using L1 products shows a remaining bias of $2.3 \mathrm{~m}$ for both $\mathrm{S}-1 \mathrm{~A}$ and $\mathrm{S}-1 \mathrm{~B}$. Note, that these results are consistent with other previous studies [7, 8], in which similar values were obtained. This remaining azimuth bias for L1 products 
corresponds to an azimuth shift of about half the pulse repetition interval. As the proposed method doesn't perform SAR azimuth processing, it is assumed that such an azimuth bias is artificial and probably arises due to an incorrect azimuth shift during operational SAR processing.

\section{CONCLUSION}

This paper proposes an easy applicable method to verify the geometric accuracy of SAR data products (L1). The method is based on the evaluation of the range delay offset and the azimuth shift derived from SAR raw data (L0 products). The geometric accuracy of both ESA's Sentinel SAR satellites (S-1A and S-1B) is determined from acquisitions over the DLR calibration field using rotatable, remote controlled corner reflectors with precisely, GPS surveyed phase center positions.

By applying the proposed method, the propagation path of each SAR pulse is completely retrieved: from transmitting the SAR pulses up to receiving the backscatter power from the point target. To estimate the range delay offset and the azimuth shift a simple pulse compression is applied in range and a phase correlation in azimuth.

As the proposed method doesn't perform a full SAR azimuth processing, the geometric offsets based on L1 and L0 products are completely independently derived. An additional effort is provided from their comparison: differences between the geometric offsets can be assumed to be artificially introduced by a processing bias or a geometric related offset like the distance between SAR antenna phase center and spacecraft's center of mass.

Results show that the azimuth bias of $2.3 \mathrm{~m}$ obtained from the analysis of L1-products is reduced to about $15 \mathrm{~cm}$ using the L0 data and the proposed method. This indicates an artificial bias is introduced by the current Sentinel-1 SAR processor. The remaining range offset of $-1.0 \mathrm{~m}$ which results for both Sentinels from the L0 data analysis is interpreted as the offset between the phase center of the SAR antenna and the spacecraft's center of mass. Assuming that such offset is already considered during SAR processing, the remaining azimuth offset derived from the L1 products is of $20 \mathrm{~cm}$, except for S-1B S1 beam which has an additional range bias of $1.6 \mathrm{~m}$.

The pixel localization accuracy has been estimated for both Sentinels by the evaluation of Stripmap mode data acquired within two different observation periods. The accuracy for the azimuth offset is derived to be of $14 \mathrm{~cm}$ for both satellites; for the range offset $12 \mathrm{~cm}$ are determined for $\mathrm{S}-1 \mathrm{~A}$ and $6 \mathrm{~cm}$ for $\mathrm{S}-1 \mathrm{~B}$. It has to be noted that the Sentinel-1 geometric requirements are already fulfilled by the L1 product results, but the current method helps to verify and improve the geometric accuracy, nevertheless.

\section{ACKNOWLEDGMENT}

The work in this paper was partially funded by the ESA Contract no. 4000119795/17/I-BG.

\section{REFERENCES}

[1] K. Ouchi "Recent Trend and Advance of Synthetic Aperture Radar with Selected Topics," Remote Sensing. 2013, 5(2), 716-807.

[2] A. Moreira, P. Prats-Iraola, M. Younis, G. Krieger, I. Hajnsek, and K P. Papathanassiou, "A tutorial on synthetic aperture radar" IEEE Geoscience and remote sensing magazine 2013, 1 (1), 6-43.

[3] Sentinel-1: ESA's Radar Observatory Mission for GMES Operational Services, ESA SP-1322/1, March 2012.

[4] D. Geudtner, P. Potin, R. Torres, P. Snoeij, D. Bibby, "Overview of the GMES Sentinel-1 Mission", In Proceedings of the 9th European Conference on Synthetic Aperture Radar, Nuremberg, Germany, 2326 April 2012; pp. 159-161.

[5] A. Schubert, D. Small, N. Miranda, D. Geudtner, and E. Meier, "Sentinel-1A/B Combined Product Geolocation Accuracy", Remote Sensing. 2017; 9(6):607.

[6] M. Schwerdt, K. Schmidt, N. Tous Ramon, G. Castellanos Alfonzo, B.J. Döring, M. Zink, and P. Prats-Iraola, "Independent Verification of the Sentinel-1A System Calibration" IEEE Journal of Selected Topics in Applied Earth Observations and Remote Sensing, vol.PP, no.99, pp.1-14, doi: 10.1109/JSTARS.2015.2449239, 2015.

[7] A. Schubert, N. Miranda, D. Geudtner, and D. Small, "Sentinel-1A/B Combined Product Geolocation Accuracy.” Remote Sensing. 2017; 9(6):607.

[8] M. Schwerdt, K. Schmidt, N. Tous Ramon, P. Klenk, N. YagueMartinez, P. Prats-Iraola, M. Zink, and D. Geudtner, "Independent System Calibration of Sentinel-1B" Remote Sens., vol. 9, no. 6, pp. 2072-4292, doi:10.3390/rs9060511, 2017.

[9] J. Reimann, M. Schwerdt, K. Schmidt, N. Tous-Ramon, et. al. "The DLR SAR Calibration Cen-ter" in Asia-Pacific Conference on Synthetic Aperture Radar (APSAR), Singapure, 2015. 